\title{
AN EXAMPLE OF NONEXCISIVENESS IN SHEAF COHOMOLOGY
}

\section{SATYA DEO}

ABSTRACT. An example is constructed to show that the conditions of tautness, besides others, imposed on spaces in a result on $\phi$-excisive couples obtained by Bredon [1] are needed.

Introduction. Let $X_{1}, X_{2}$ be a pair of subspaces of a topological space $X$, and $\phi$ a family of supports on $X$. Bredon [1, Definition 13.1, p. 65] calls the pair $\left(X_{1}, X_{2}\right) \phi$-excisive with respect to sheaf-theoretic relative cohomology groups, defined by him [1, p. 57], if the inclusion map $i:\left(X_{1}, X_{1} \cap X_{2}\right) \rightarrow\left(X_{1} \cup X_{2}, X_{2}\right)$ induces isomorphism

$$
i^{*}: H_{\phi \cap\left(x_{1} \cup X_{2}\right)}^{*}\left(X_{1} \cup X_{2}, X_{2} ; \mathbb{Q}\right) \rightarrow H_{\phi \cap X_{1}}^{*}\left(X_{1}, X_{1} \cap X_{2} ; \mathbb{Q}\right)
$$

for any sheaf $\mathbb{Q}$ on $X$. Among other things he has proved the following

Proposition [1,13.3, p. 68]. Let $X=X_{1} \cup X_{2}$ and $A=X_{1} \cap X_{2}$. If $\phi$ is a family of supports on $X$ with

$$
\left(\phi \cap X_{1}\right)\left|X_{1}-A=\phi\right|\left(X-X_{2}\right) \subset \phi \mid \operatorname{Int}\left(X_{1}\right)
$$

and such that $X_{2}$ is $\phi$-taut and $A$ is $\left(\phi \cap X_{1}\right)$-taut in $X_{1}$, then $\left(X_{1}, X_{2}\right)$ is $\phi$-excisive.

and a consequent,

Corollary. If $X=X_{1} \cup \operatorname{Int}\left(X_{2}\right)=\operatorname{Int}\left(X_{1}\right) \cup X_{2}$ and $X_{1}, X_{2}, X_{1} \cap X_{2}$ are all $\phi$-taut in $X$, then $\left(X_{1}, X_{2}\right)$ is $\phi$-excisive.

At the end of the proof he raises a problem: Are the tautness conditions in the above Proposition and Corollary needed? We give an example to show that the answer to the case of the Proposition is affirmative. The case of the Corollary is still open. His result is of interest not only for sheaf cohomology, but it also enlarges the class of excisive couples [2, p. 188] for Alexander-Spanier cohomology, as we shall see later, and consequently,

Received by the editors July 11, 1973 and, in revised form, J anuary 10, 1974. AMS (MOS) subject classifications (1970). Primary 55B99; Secondary 55B30. Key words and phrases. Sheaves, family of supports $\phi, \phi$-tautness. 
the validity of Mayer-Vietoris exact sequences. Our example uses a sheaf of abelian groups as the simple sheaf $\mathbf{R}$ of real numbers on a metric space $X$. For all definitions, notations and results indirectly involved, [1] is our complete reference.

Example. Let $X=\mathbf{R}^{2}$ be the 2-Euclidean space and $\mathbf{R}$ be the constant sheaf of real numbers on $X$. Define

$$
\begin{aligned}
& X_{1}=\left\{(x, y) \mid x^{2}+y^{2} \leq 1 \text { or } y \geq 0\right\}, \\
& X_{2}=\left\{(x, y) \mid x^{2}+y^{2}>1 \text { and } y \leq 0\right\} \cup\{(1,0),(-1,0)\}, \\
& D=\left\{(x, y) \mid x^{2}+y^{2}<1\right\}, \\
& B=\left\{(x, y) \mid x^{2}+y^{2}=1 \text { and } y<0\right\}, \\
& \phi=c l d \mid X-(D \cup B) .
\end{aligned}
$$

First of all let us observe that the family $\phi$ of supports is not paracompactifying because the closed line segment $[(0,1),(0,2)] \epsilon \phi$ but has no neighborhood which is a me mber of $\phi$. Since $X_{1}$ is closed, it is clear that $(*)$ holds. It may be noted that $\phi \cap X_{1}=\phi \mid X_{1}$ and $\phi \cap A=\phi \mid A$. Now since $H_{\phi}^{0}(X)$ is simply the group of those real valued functions which are locally constant and have their support in $\phi$, and because $X$ is connected, $H_{\phi}^{0}(X)=0$. By a similar argument, $H_{\phi \cap X_{2}}^{0}\left(X_{2}\right)=0=H_{\phi \cap X_{1}}^{0}\left(X_{1}\right)$. But, since $A \in \phi$ and has two components, $H_{\phi \cap A}^{0}(A)=\mathbf{R} \oplus \mathbf{R}$.

Next we claim that $X$ is $\phi$-contractible to any point of $D \cup B$. To see why it is $\phi$-contractible to the origin $(0,0)$, consider the $\phi$-proper maps $I_{X}$ and the constant map $C: X \rightarrow X$ with value $(0,0)$. Define the usual homotopy $F: X \times I \rightarrow X$ by $F((x, y), t)=((1-t) x,(1-t) y)$. Then for any $K \in \phi$,

$$
F^{-1}(K)=\{((x, y), t) \mid((1-t) x,(1-t) y) \in K\}=\bigcup_{0 \leq t<1} S_{t} \times t,
$$

where

$$
S_{t}=\{(x, y) \mid(x, y) \in K /(1-t)\} \text {. }
$$

This is a closed subset of $S \times I \in \phi \times I$, where $S$ is the union of all rays starting from each point of $K$ away from the origin. Hence by the homotopy axiom for constant sheaves [1, Theorem 11.4, p. 56], $H_{\phi}^{n}(X)=0$ for every $n \geq 0$.

Finally, one observes that $\left(X_{1}, X_{2}\right)$ cannot be $\phi$-excisive because the Mayer-Vietoris sequence $[1$, p. 68$]$

$$
0 \rightarrow H_{\phi}^{0}(X) \rightarrow H_{\phi \cap X_{1}}^{0}\left(X_{1}\right) \oplus H_{\phi \cap X_{2}}^{0}\left(X_{2}\right) \rightarrow H_{\phi \cap A}^{0}(A) \rightarrow H_{\phi}^{1}(X) \rightarrow \cdots
$$


Now to see that $A$ is not $\phi \cap X_{1}$-taut in $X_{1}$, consider the locally constant function $f: A \rightarrow R$ with any nonzero value, which is in fact an element of $H_{\phi \cap A}^{0}(A)$. Then this has no extension $f^{\prime}$ to a neighborhood $N$ of $A$ in $X_{1}$ which has its support in $\phi \cap N$, i.e.,

$$
\eta: \lim _{\rightarrow} H_{\phi \cap N}^{0}(N) \rightarrow H_{\phi \cap A}^{0}(A)
$$

is not epic, and our assertion follows from [1, Theorem 10.5, pp. 52-53].

We are unable to see whether or not each of the tautness conditions is separately needed.

Remark. The conclusion that $\left(X_{1}, X_{2}\right)$ is not $\phi$-excisive depends solely on the fact that $\phi$ is not a paracompactifying family of supports, since otherwise $X_{1}, X_{2}$ being arbitrary subsets of a metric space would be $\phi$-taut. For example, when $\phi=\mathrm{cl} d$, all the groups are Alexander-Spanier cohomology groups, and in that case $\left(X_{1}, X_{2}\right)$ is $\phi$-excisive by the Proposition itself. This very last statement illustrates the situation about excisive couples not covered under the excisive couples of Alexander-Spanier or Čech cohomology theories.

The author is thankful to Professor John W. Keesee for many useful discussions.

\section{REFERENCES}

1. Glen E. Bredon, Sheaf theory, McGraw-Hill, New York, 1967.

2. Edwin H. Spanier, Algebraic topology, McGraw-Hill, New York, 1966. MR 35 \# 1007.

DEPARTMENT OF. MATHEMATICS, UNIVERSITY OF ARKANSAS, FAYETTEVILLE, ARK ANSAS 72701

Current address: Department of Mathematics, Allahabad University, Allahabad, 211002, India 